$16^{\text {th }}$ International Congress of Metrology, 14009 (2013)

DOI: $10.1051 /$ metrology/201314009

(C) Owned by the authors, published by EDP Sciences, 2013

\title{
EMRP-ENV04: Traceable radiometry for remote measurement of climate parameters
}

\author{
Romain Etienne ${ }^{1, \mathrm{a}}$ et Jimmy Dubard ${ }^{1}$ \\ ${ }^{1}$ LCM, LNE/CNAM, 29 avenue Roger Hennequin, 78197 TRAPPES, France
}

\begin{abstract}
Remote sensing of the Earth from space, utilising the full electromagnetic spectrum, is the only means of obtaining the global data needed to underpin climate change research and provide the knowledge to enable policy makers to adopt appropriate mitigation and adaptation strategies. In some cases changes of a few tenths of a percent per decade are required relying on measurands that it is currently only possible to carry out NMIs (National Metrology Institute) laboratories at similar levels of uncertainty. A "virtual" centre for EO (Earth Observation) traceability is needed to lead international efforts to develop calibration and validation standards and techniques, pre \& post launch, for land, ocean \& atmosphere to extend the capabilities of the SI to meet the needs of climate measurement.
\end{abstract}

\section{Introduction}

The Earth's climate is undoubtedly changing; however, the time scale, consequences and causal attribution remain the subject of significant debate and uncertainty. Detection of subtle indicators from a background of natural variability requires measurements over a time base of decades. This places severe demands on the instrumentation used, requiring measurements of sufficient accuracy and sensitivity that can allow reliable judgements to be made decades apart. The International System of Units (SI) and the network of National Metrology Institutes were developed to address such requirements. However, ensuring and maintaining SI traceability of sufficient accuracy in instruments orbiting the Earth presents a significant new challenge to the metrology community. There is some key measurands and applications driving the uncertainty demand of the climate community in the solar reflective domain, e.g. solar irradiances and reflectances/radiances of the Earth. This project describes a new satellite mission, called TRUTHS (Traceable Radiometry Underpinning Terrestrial and Helio Studies), which enables, for the first time, highaccuracy SI traceability to be established in orbit. The direct use of a 'primary standard' and replication of the terrestrial traceability chain extends the SI into space, in effect realizing a 'metrology laboratory in space'. We present the objectives of the project and the work to be performed. We focus on LNE contributions particularly to workpackages (WP):

- WP4 task 4.1 : Design and test prototype optical fibre based spectral radiometric delivery system for in-flight calibration system of TRUTHS

\footnotetext{
${ }^{\mathrm{a}}$ romain.etienne@lne.fr
}

\section{ENV04 project}

The overall concept of the ENV04 project can be summarized as: TRUTHS, a mission to measure, SItraceably and with an unprecedented accuracy, the interaction of solar radiation with the Earth as a benchmark reference to enable the detection of decadal climate change. TRUTHS will also enhance the performance and traceability of other EO systems to deliver additional societal benefits, and, in some cases, climate quality data product, form existing instruments. The project is coordinated by the NPL and involves 12 participants (NPL, AALTO, INRIM, JRC, LNE, MIKES, PTB, SFI Davos, BUW, DLR, FGI and FZJ)

\subsection{WP1: Pre-flight laboratory based transfer calibration standards and methodologies}

Calibration and characterisation of measurement instrumentation traceable to SI units before use, is a fundamental principle of metrology and is adhered to by all sectors including the Earth Observation community. In many industrial and terrestrial based applications the maturity of this philosophy has led to well-defined methodologies, documentary standards and robust, often generic transfer standards to enable a hierarchical calibration and delivery chain to be established company calibration laboratories, linked to accredited laboratories in turn linked to national metrology institutes. There are often a multitude of different instruments capable of measuring similar parameters. Some require bespoke tailored calibration facilities to be designed and operated, within production lines for example. However, in most cases it has been possible for 
NMIs, at the top of the traceability chain, to establish reference standards and methods that can generically serve a wide range of users in many different sectors.

This WP will :

-develop, evaluate and transfer to stakeholders procedures for the characterisation and correction of stray-light characteristics for hyperspectral imaging spectroradiometers by using a tuneable laser-based source and apply these to the airborne sensor ROSIS (Reflective Optics System Imaging Spectrometer).

-develop and apply a large aperture homogenous radiance source for the linearity and radiometric calibration of wide field of view airborne sensors.

-develop techniques and standards to allow the spectrally resolved radiometric calibration of space borne sensors under vacuum.

-establish a prototype European capability for the calibration of microwave sounders.

\subsection{WP2: In-flight on-board transfer standards}

The purpose of this WP is the development, characterization and application of a series of large aperture on-board radiance standards for the in-flight calibration of imaging IR spectroradiometers used to measure atmospheric radiance for climate variables such as temperature fields, cloud parameters, aerosols, water vapour, ozone and other trace species by e.g. limb sounding. The output of this WP will enable significant improvements to be made to the calibration strategies of future IR hyperspectral imagers, and indeed other IR sensors and ensure that SI traceable uncertainties now demanded by the climate community can be achieved. The uncertainty requirement is highly demanding with the following standard uncertainties being the target: -uncertainty of absolute radiance measurement: $u=0.01$ -uncertainty of relative radiance measurement (spatially and spectrally): $u=0.001$ ( 0.0025 threshold).

This WP will develop the metrological capability to radiometrically characterise a new set of radiance standards for the on-board calibration of mid infrared atmospheric imagers at uncertainty levels needed for climate studies. This will also lead to improved, fit for purpose designs tailored to a range of current and future applications and thus serving as an example of the value of this JRP to the atmospheric imaging community.

\section{This WP will :}

-specify and validate the radiometric capabilities needed at the NMIs for characterizing IR "on-board" calibration standards suitable for imaging spectro-radiometers.

-characterize radiance standards for aircraft-borne hyperspectral imagers.

-investigate the applicability of the radiance standards for aircraft-borne hyperspectral imagers.

-develop and characterize a large aperture calibration standard for balloon-borne hyperspectral imagers. -design a large aperture calibration standard for a satellite-based on-board calibration of a hyperspectral imager.

\subsection{WP3: Recovering/establishing in-flight traceability through reference standard measurements and test-sites}

As climate is the key driver for this project, this WP concentrates on radiometric test-sties in support of sensors measuring ECVs: Ocean colour (OC), in particular; water-leaving radiances, and those used for land viewing sensors. In the latter case, effort will mostly address automation and maintenance of traceability of measurements over a distributed surface area. The results of this will not only be applicable to $\mathrm{L} 1$ radiances (radiometric gains) using bright desert targets but also vegetated sites for both L2 products and also as darker targets for linearity checks on sensors and of course those used for $\mathrm{OC}$ as well.

This WP will :

-reduce uncertainty in Ocean Colour (OC) through establishment of rigorous SI traceability.

-establish SI traceable validation of the World's Radiative Transfer models/codes and improve their performance though provision of key input parameters.

-do autonomous SI traceable measurements of geographically distributed surface spectral reflectance/radiance for reference test sites.

\subsection{WP4: A "standard laboratory in space" for benchmark measurements of climate}

Although this WP and its activities have the demonstration of "in-flight" SI traceable calibration methodology of TRUTHS as its driving requirement, many of the concepts and associated instrumentation have application terrestrially. This is particularly true for pre-flight calibration of optical imagers (WP1), which needs to be carried out in vacuum and increasingly at uncertainties commensurate with those obtainable in the primary calibration facilities of national metrology institutes i.e. not surprisingly the same drivers as TRUTHS. Thus whilst for terrestrial implementations some of the specific constraints are reduced, the need to transfer primary scales from an NMI to a dedicated space calibration facility will also benefit from the outputs of this WP. In scoping the technical work programme of this WP it should be noted that the primary standard cryogenic radiometer which is the heart of the calibration methodology has already been designed and constructed through a consortium of NMIs: NPL, SFI Davos (PMOD) and Metas. This Cryogenic Solar Absolute Radiometer (CSAR) also has a primary terrestrial application, the measurement of ground-level Total Solar Irradiance (TSI), ultimately with a view to be a replacement of the world standard group of radiometers, the current World Radiometric Reference of the WMO. CSAR will be provided for use in this project as required. 
This WP will :

-design and test prototype optical fibre based spectral radiometric delivery system for in-flight calibration system of TRUTHS.

-design and test of prototype Polarising Transfer Radiometer (PTR) for TRUTHS in-flight calibration system and for establishing traceability in vacuum for pre-flight calibration of optical sensors.

-characterise and demonstrate performance of CSAR as primary standard for TRUTHS and for measurements of total solar irradiance.

-demonstrate end-to-end calibration methodology of TRUTHS.

\section{LNE contribution to ENV04 project}

LNE is involved in WP4. It is charge of task 4.1 consisting to design, optimise and characterise a prototype fibre optic delivery system for in-flight spectroradiometric calibration of TRUTHS. Figure 1 shows a concept of TRUTHS satellite with the optical fibre transfer system.

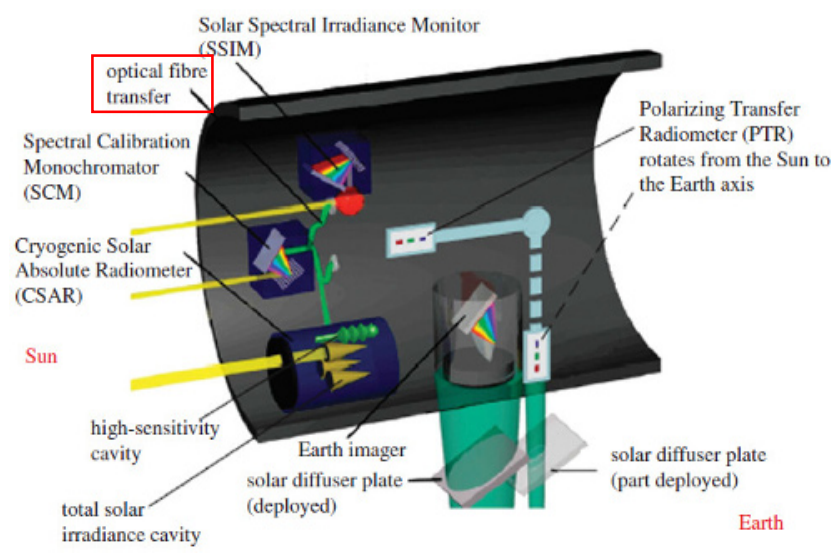

Figure 1. TRUTHS satellite concept

One of the key elements within the calibration strategy of TRUTHS is the need to transfer monochromatic optical radiation from the Sun, dispersed by a monochromator, between the entrance ports of various optical instruments. The transfer mechanism needs to have high throughput over a wide spectral range (200 to $2450 \mathrm{~nm}$ ) and be radiometrically stable $(<0.1 \%)$ between sequential instrument to instrument measurements and be suitable for space-flight.

The key challenges within this task are to design, characterise and optimise a system, including the radiation coupling (input and output), to enable a full "lab-based" demonstration of the TRUTHS calibration concept, from primary standard to spectrometer in vacuum. The breadboard evaluation will enable full detailed performance analysis to be carried out in readiness for a future mission. In addition to considerations on high throughput, are radiation damage, and losses due to changes in bending and thermal environment etc...

\subsection{Requirements specification}

The main generic requirements are :

-Optical fibre delivery system needs to enable maximum transfer of radiation from monochromator exit slit to entrance aperture of instruments

-Special fibre arrangement inside the bundle. That mean, fibres at the center of the rectangular input (interface to the monochromator) should be at the center of the circular output (interface to the instruments under test) -No movement induced losses $(<0.05 \%)$ (maximum linear movement between instruments $30 \mathrm{~cm}$ )

-Vacuum compatible

-Wide spectral range 200 to $2450 \mathrm{~nm}$ (maximum 3 independent systems)

Figure 2 provides an example of how the optical fibre delivery system is anticipated to interface to the monochromator of TRUTHS.

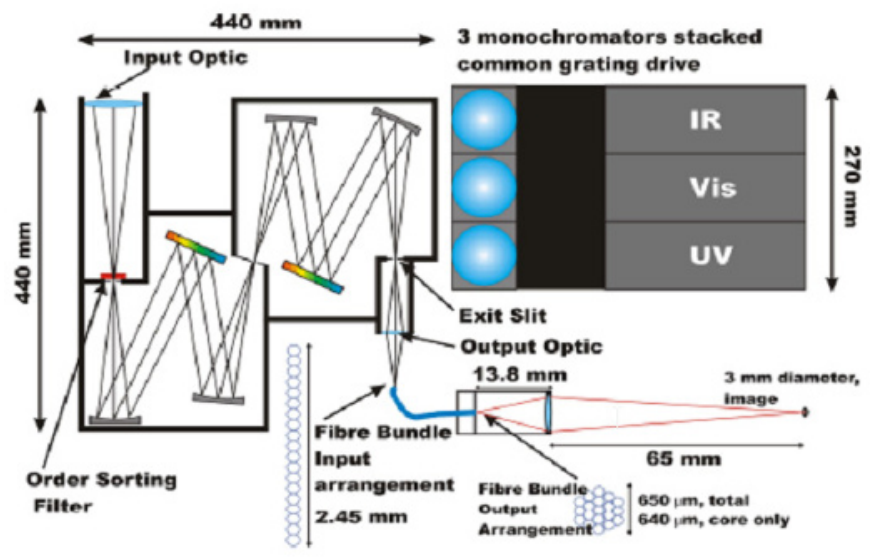

Figure 2. Drawing of the interface

Two fibre bundles were ordered, one for UV/Vis spectral range $(200 \mathrm{~nm}-1000 \mathrm{~nm})$ and one for IR spectral range $(1000 \mathrm{~nm}-2500 \mathrm{~nm})$. They have the following characteristics :

-Type of fibre

-FG200UCC multimode for the UV/Vis bundle

-BFL22 22 multimode for the IR bundle

-High throughput $>70 \%$

-Hard shielding to prevent damage from radiation in space

-Special fibre arrangement

-Input bundle : rectangular 6 columns, 41 rows

-Output bundle : circular

-Diameter of the fibre : $240 \mu \mathrm{m}$ (core: $200 \mu \mathrm{m}$ )

-Numerical aperture : 0.22 (f number 4.7)

-Number of fibres : 246

-Length of the bundle : $500 \mathrm{~mm}$

-Glue : ND353 (space compatible)

-Metallic jacket

-Fitted with optics to get $5 \mathrm{~mm}$ diameter beam at $120 \mathrm{~mm}$ from output

Figure 3 shows a picture of the two different bundles. 

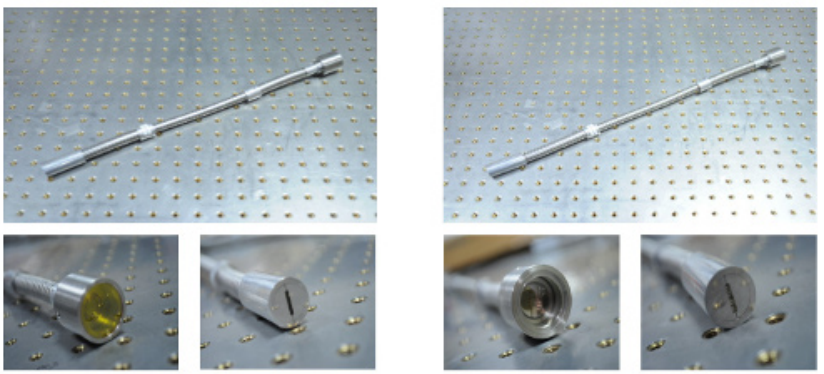

Figure 3. IR bundle on the left pictures, UV/Vis bundle on the right pictures

\subsection{First observations of the fibre bundles}

The first test was to observe extremities of the bundles using a microscope (binocular) in order to check the optical fibres number and the surface quality of the input and output.
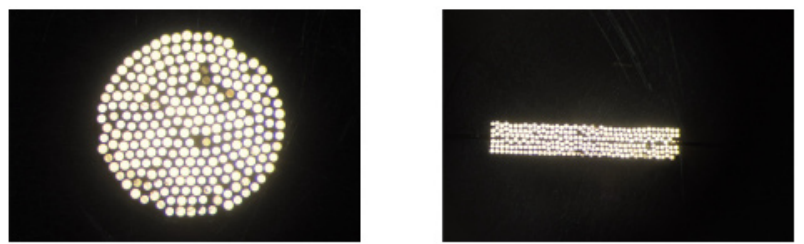

Figure 4. UV/Vis bundle

Observing the UV/Vis bundle we can see 241 fibres "on"
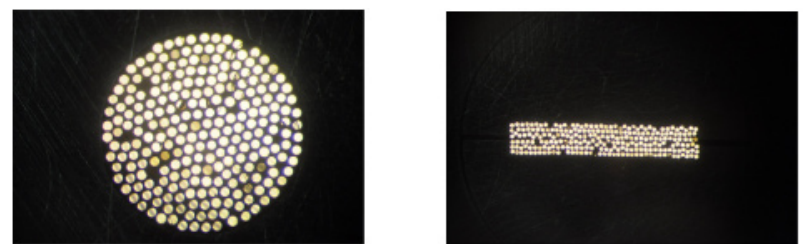

Figure 5. UV/Vis bundle

Observing the Vis/IR bundle we can see 249 fibres "on"

Maybe, the fibres "off" are broken or covered with glue like we can see on the yellow one. Bundles were returned to the manufacturer for an extra polishing of the extremities.

The second test was to observe the special fibre arrangement inside the bundle. For this test, the bundle was mounted on a motorized positioner. The rectangular input was positioned in front of the exit slit of a monochromator. Moving the bundle with a step of 0.1 $\mathrm{mm}$ and we observed the output image formed by the lens on a screen.

The following figures (figure 6a to 6e) describe step by step this observation test.
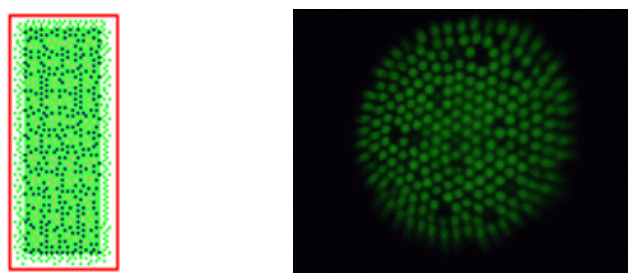

Figure 6a
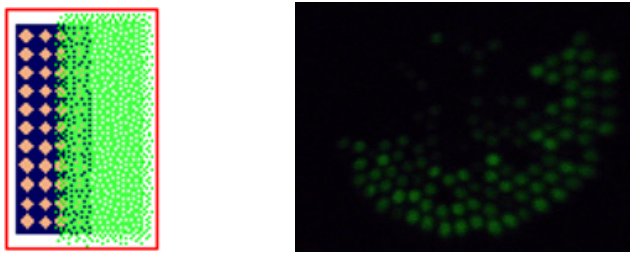

Figure 6b
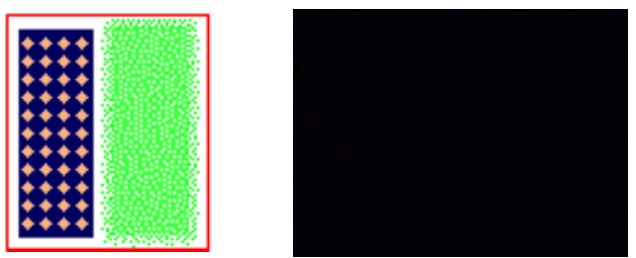

Figure 6c
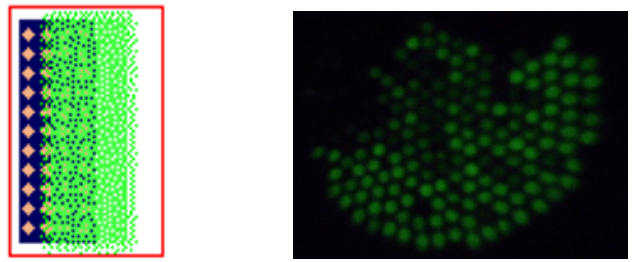

Figure 6d
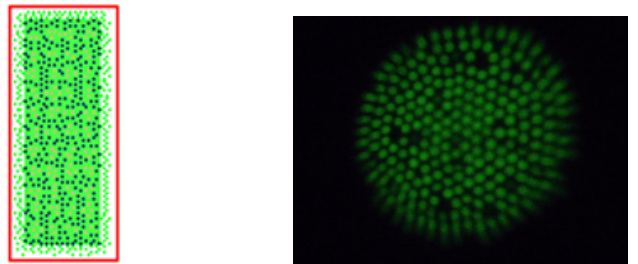

Figure 6e

The special fibre arrangement inside each bundle is correct. The fibres of the columns at the center of the rectangular input are at the center of the circular output.

\section{Conclusion}

The aim of the ENV04 is the TRUTHS mission offering a novel approach to the provision of key scientific data with unprecedented radiometric accuracy for Earth Observation (EO) and solar studies and in particular for decadal climate change. LNE contributes to the realization of the prototype fibre optic delivery system for in-flight spectroradiometric calibration of TRUTHS and its optimization and characterization. 
In conclusion of these first observation tests:

-some fibres are broken or obstructed on each bundle.

-the surface quality of the input and output of the fibre

bundles are not perfect (glue residue...)

-need optical tests to know if these points are critical or not

-additional polishing of fibre bundles connectors

-special fibre arrangement is good.

Next steps are to achieve the optical characterization (spectral transmission of each bundle) and the mechanical tests (optical stability).

\section{Acknowledgements}

The EMRP is jointly funded by the EMRP participating countries within EURAMET and the European Union

\section{References}

1. Nigel Fox, EMRP 2010 JRP 17e "Traceable Radiometry for Remote measurement of Climate Parameters".

2. Nigel Fox, Andrea Kaiser-Weiss, Werner Schmutz, Kurtis Thome, Dave Young, Bruce Wielicki, Rainer Winkler and Emma Woolliams "Accurate radiometry from space: an essential tool for climate studies", The Rotal Society (2011) 